\title{
Gas Content in the GMC G333: hierarchical structure of turbulence
}

\author{
N. Lo ${ }^{1}$, M. R. Cunningham ${ }^{2}$, P. A. Jones ${ }^{2}$ and L. Bronfman ${ }^{1}$ \\ ${ }^{1}$ Departamento de Astronomía, Universidad de Chile, Camino El Observatorio 1515 \\ Las Condes, Santiago, Chile. email: nlo@das.uchile.cl \\ ${ }^{2}$ School of Physics, University of New South Wales, Sydney, Australia
}

\begin{abstract}
We present our preliminary results on the application of dendrogram statistics to the carbon monoxide PPV map of the giant molecular cloud G333. We obtain the dendrograms at various merging levels and found the clustering of branches is independent from the merging levels. The statistics of intensity distributions show gravity is possibly significant in this cloud and the gas may be sonic. Application of this method to other molecular lines data are required for further analysis of the cloud properties.
\end{abstract}

Keywords. ISM: clouds - ISM: kinematics and dynamics — turbulence

Turbulence is able to create the hierarchical structure of molecular clouds, over the past decades various techniques have been developed to study turbulence in the ISM, one such method is dendrogram (tree diagram). Burkhart et al. (2012) demonstrates by applying dendrogram statistics to synthetic observations of isothermal MHD turbulence, it is possible to retrieve the physical mechanism behind ISM hierarchical structure. Here we present our preliminary result when this method is applied to ${ }^{13} \mathrm{CO}$ PPV map of the giant molecular cloud complex G333 (Bains et al. 2006, Wong et al. 2008, Lo et al. 2009). We construct the dendrograms at various intensity merging levels $(\delta)$, to explore how the dendrogram changes at different $\delta$ values on observed data. Fig. 1 (left and middle) are dendrograms at various $\delta$ levels (here we present two only). As we increase the $\delta$ level, the number of leave decreases, however, it is also clear that the clustering shape of leave and branches does not change. This gives us confidence that the tree property does not heavily depend on the $\delta$ value. We derived the first four moments of the distributions at each of the $\delta$ level (Fig. 1 right). Instead of a gradual decrease in kurtosis, it shows a rather steep drop to near zero below $\delta=1$. As the $\delta$ value increases, more weaker and larger scale structures are merged with the brighter features, and thus the kurtosis decreases. If the gas distribution is disperse in general, then the kurtosis should stays more or less constant. This rapid drop possibly suggests gravity is significant and the gas may be sonic. The significance of $\delta=1\left(T_{\mathrm{MB}}=1.8 \mathrm{~K}\right)$ is yet to be investigated.

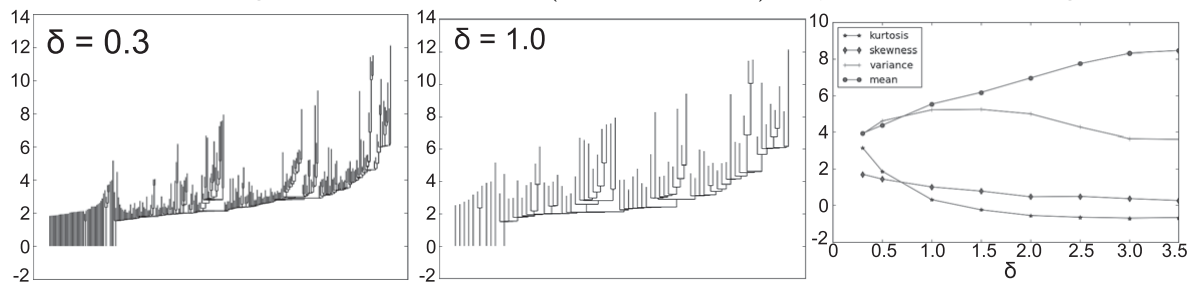

Figure 1. Dendrogram of the G333 13CO PPV map and distribution statistics.

\section{References}

Bains, I., Wong, T., Cunningham, M. et al. 2006, MNRAS, 367, 1609

Burkhart, B., Lazarian, A., Goodman, A., \& Rosolowsky, E. 2012, ApJ, submitted

Lo, N., Cunningham, M. R., Jones et al. 2009, MNRAS, 395, 1021

Wong, T., Ladd, E. F., Brisbin, D. et al. 2008, MNRAS, 386, 1069 